\title{
Conformal Invariance and Conserved Quantity of Hamilton System under Second-Class Mei Symmetry
}

\author{
JIAN-LE CAI* \\ College of Science, Hangzhou Normal University, Hangzhou 310018, China
}

(Received October 28, 2009)

\begin{abstract}
Conformal invariance and conserved quantities of Hamilton system under second-class Mei symmetry are studied. The single-parameter infinitesimal transformation group and infinitesimal transformation vector of generator are introduced. The definitions about conformal invariance of Hamilton function and conformal invariance of Hamilton system under second-class Mei symmetry are given. The relationship between the system's conformal invariance and Mei symmetry are discussed. The necessary and sufficient condition that the system's conformal invariance would be Mei symmetry is deduced. The system's corresponding conserved quantities are obtained with the aid of a structure equation which is satisfied by the gauge function. Lastly, an example is provided to illustrate the application of the result.
\end{abstract}

PACS numbers: 02.20.Sv, 11.30.-j

\section{Introduction}

Since Noether, a German woman scientist, published her famous paper "Invariante Variationsprobleme" in 1918 [1], the theory of symmetries and conserved quantities has played an important role in mathematics and physics [2, 3], especially in modern analytical mechanics. From then on, the study on the Noether symmetry has gradually progressed to become mature [4]. In 1979, Lutzky applied the Lie theory to the differential equations of motion for a mechanical system, and obtained a new type of conserved quantities of the dynamical system under Lie point symmetrical transformations, i.e. the Lutzky conserved quantities [5]. In the beginning of this century, Mei presented a new symmetry - the form invariance [6]. Mei's form invariance is also called Mei symmetry [7-15], which means that the dynamical function in the equation of motion still satisfies the equation's primary form after some infinitesimal transformations. Then more and more attention has been paid to research on Mei symmetry and conserved quantity, which was quickly extended to all types of mechanical systems [4].

In the past few years, some important results on symmetry and conserved quantity for the Hamilton system have been gained [16-24]. In Refs. [25-29], researchers have studied the Mei symmetry and conserved quantity for the Hamilton system. Recently, we have discussed the conformal invariance and conserved quantities of the Lie symmetry for Lagrange system, Hamilton system and general holonomic mechanical system [30-32], and of the Mei symmetry for Lagrange system and general holonomic mechanical system $[33,34]$. However, till now the research on conformal invariance of Mei symmetry for

\footnotetext{
* e-mail: caijianle@yahoo.com.cn
}

Hamilton system has not been reported yet. Thus, conformal invariance and conserved quantity for Hamilton system under second-class Mei symmetry are studied in the paper. An example is given to illustrate their applications.

\section{Second-class Mei symmetry of Hamilton system}

Here we study a mechanical system of $N$ particles, with its configuration determined by $n$ generalized coordinates $q_{s}(s=1, \ldots, n)$. The Hamilton canonical equations of the system can be written as

$$
\dot{q}_{s}=\frac{\partial H}{\partial p_{s}}, \quad \dot{p}_{s}=-\frac{\partial H}{\partial q_{s}}, \quad s=1, \ldots, n,
$$

where $q_{s}$ and $p_{s}$ are the generalized coordinates and generalized momenta, respectively. They are also called canonical variables.

We introduce a single-parameter infinitesimal transformations group for time $t$, canonical variables $q_{s}$ and $p_{s}$

$$
\begin{aligned}
& t^{*}=t+\Delta t, \quad q_{s}^{*}\left(t^{*}\right)=q_{s}(t)+\Delta q_{s}, \\
& p_{s}^{*}\left(t^{*}\right)=p_{s}(t)+\Delta p_{s} .
\end{aligned}
$$

Their expanding forms are

$$
\begin{aligned}
& t^{*}=t+\varepsilon \xi_{0}(t, \boldsymbol{q}, \boldsymbol{p}), \quad q_{s}^{*}\left(t^{*}\right)=q_{s}(t)+\varepsilon \xi_{s}(t, \boldsymbol{q}, \boldsymbol{p}), \\
& p_{s}^{*}\left(t^{*}\right)=p_{s}(t)+\varepsilon \eta_{s}(t, \boldsymbol{q}, \boldsymbol{p}),
\end{aligned}
$$

where $\xi_{0}, \xi_{s}$ and $\eta_{s}$ are called the infinitesimal generators of $t, q_{s}$ and $p_{s}$, respectively, under the infinitesimal transformations $(2)$, and $\varepsilon$ is a small parameter. We introduce an infinitesimal transformation vector of generator

$$
X^{(0)}=\xi_{0} \frac{\partial}{\partial t}+\xi_{s} \frac{\partial}{\partial q_{s}}+\eta_{s} \frac{\partial}{\partial p_{s}},
$$

under the single-parameter infinitesimal transformations (3), Hamilton functions $H$ become $H^{*}$, so we can write 


$$
\begin{aligned}
H^{*} & =H\left(t^{*}, \boldsymbol{q}^{*}, \boldsymbol{p}^{*}\right) \\
& =H(t, \boldsymbol{q}, \boldsymbol{p})+\varepsilon X^{(0)}(H)+\mathrm{O}\left(\varepsilon^{2}\right) .
\end{aligned}
$$

Definition 1. If the form of Hamilton system dynamic Eq. (1) remains unchanged under the infinitesimal transformations (3), i.e.

$$
\dot{q}_{s}^{*}=\frac{\partial H^{*}}{\partial p_{s}}, \quad \dot{p}_{s}^{*}=-\frac{\partial H^{*}}{\partial q_{s}}, \quad s=1, \ldots, n,
$$

then this kind of invariance is called the second-class Mei symmetry of Hamilton system.

Taking the derivatives of $q_{s}^{*}$ and $p_{s}^{*}$ with respect to time in Eq. (3), and substituting Eq. (5) and (1) into Eq. (6), neglecting higher-order $\varepsilon^{2}$ and above the lesser term, we can obtain the determining equation of second-class Mei symmetry for Hamilton system

$$
\begin{aligned}
& \left.\left\{\dot{\xi}_{s}-\frac{\partial}{\partial p_{s}}\left[X^{(0)}(H)\right]\right\}\right|_{\dot{q}_{s}=\frac{\partial H}{\partial p_{s}}}=0, \\
& \left.\left\{\dot{\eta}_{s}+\frac{\partial}{\partial q_{s}}\left[X^{(0)}(H)\right]\right\}\right|_{\dot{p}_{s}=-\frac{\partial H}{\partial q_{s}}}=0 .
\end{aligned}
$$

\section{Conformal invariance of second-class Mei symmetry for Hamilton system}

Definition 2. For the Hamilton function $H$, if there is a product factor $M$ satisfying the following condition:

$$
X^{(0)}(H)=M H,
$$

then this kind of invariance is called the conformal invariance of the Hamilton function $H$ under the single-parameter infinitesimal transformations (3). Equation (8) is the determining equation of conformal invariance of Hamilton function $H$, where $M$ is called conformal factor.

Definition 3. For the Hamilton Eqs. (1), if there are matrices $\tilde{P}_{s}^{k}$ and $\tilde{Q}_{s}^{k}$ satisfying the following condition:

$$
\begin{gathered}
\dot{\xi}_{s}-\frac{\partial}{\partial p_{s}}\left\{X^{(0)}(H)\right\}=\tilde{P}_{s}^{k}\left(\dot{q}_{k}-\frac{\partial H}{\partial p_{k}}\right), \\
\dot{\eta}_{s}+\frac{\partial}{\partial q_{s}}\left\{X^{(0)}(H)\right\}=\tilde{Q}_{s}^{k}\left(\dot{p}_{k}+\frac{\partial H}{\partial q_{k}}\right), \\
s, k=1, \ldots, n,
\end{gathered}
$$

then this kind of invariance is called the conformal invariance of Eq. (1) under the single-parameter infinitesimal transformations (3). Equations (9) are the determining equations of conformal invariance for the second-class Mei symmetry of Hamilton system (1), where $\tilde{P}_{s}^{k}$ and $\tilde{Q}_{s}^{k}$ are called conformal factors.

\section{The relationship between the conformal invariance and Mei symmetry}

Theorem 1. If the Hamilton equations show conformal invariance of second-class Mei symmetry under the single-parameter infinitesimal transformations (3), then they possess the second-class Mei symmetry.
Proof. Substitute Eq. (1) into determining Eqs. (9), we can obtain Eq. (7).

Theorem 2. For the Hamilton system (1), if the generators $\xi_{0}, \xi_{s}$ and $\eta_{s}$ of the infinitesimal transformations (3) satisfy the determining Eqs. (7) of conformal invariance for second-class Mei symmetry, and there are matrices $\tilde{\mu}_{s}^{k}$ and $\tilde{\nu}_{s}^{k}$ satisfying the following condition:

$$
\begin{aligned}
& \left\{\dot{\xi}_{s}-\frac{\partial}{\partial p_{s}}\left[X^{(0)}(H)\right]\right\} \\
& \quad-\left.\left\{\dot{\xi}_{s}-\frac{\partial}{\partial p_{s}}\left[X^{(0)}(H)\right]\right\}\right|_{\dot{q}_{s}=\frac{\partial H}{\partial p_{s}}} \\
& =\tilde{\mu}_{s}^{k}\left(\dot{q}_{k}-\frac{\partial H}{\partial p_{k}}\right), \\
& \left\{\dot{\eta}_{s}+\frac{\partial}{\partial q_{s}}\left[X^{(0)}(H)\right]\right\} \\
& -\left.\left\{\dot{\eta}_{s}+\frac{\partial}{\partial q_{s}}\left[X^{(0)}(H)\right]\right\}\right|_{\dot{p}_{s}=-\frac{\partial H}{\partial q_{s}}}, \quad s, k=1, \ldots, n,
\end{aligned}
$$

then the necessary and sufficient condition that the conformal invariance would be second-class Mei symmetry of system is

$$
\tilde{P}_{s}^{k}=\tilde{\mu}_{s}^{k}, \quad \tilde{Q}_{s}^{k}=\tilde{\nu}_{s}^{k} .
$$

Proof. In fact, if there are matrices $\tilde{\mu}_{s}^{k}, \tilde{\nu}_{s}^{k}$ satisfying Eq. (10), and the Mei symmetry of Hamilton system Eq. (1) satisfies Eq. (7), then Eq. (10) becomes

$$
\begin{aligned}
& \dot{\xi}_{s}-\frac{\partial}{\partial p_{s}}\left[X^{(0)}(H)\right]=\tilde{\mu}_{s}^{k}\left(\dot{q}_{k}-\frac{\partial H}{\partial p_{k}}\right), \\
& \dot{\eta}_{s}+\frac{\partial}{\partial q_{s}}\left[X^{(0)}(H)\right]=\tilde{\nu}_{s}^{k}\left(\dot{p}_{k}+\frac{\partial H}{\partial q_{k}}\right) .
\end{aligned}
$$

According to the definition (9), the conformal factor of the system $\tilde{P}_{s}^{k}=\tilde{\mu}_{s}^{k}, \tilde{Q}_{s}^{k}=\tilde{\nu}_{s}^{k}$.

Vice versa, according to the definition (9) and Eq. (10), we can easily verify that there exists a relationship as follows:

$$
\begin{gathered}
\left(\tilde{P}_{s}^{k}-\tilde{\mu}_{s}^{k}\right)\left(\dot{q}_{k}-\frac{\partial H}{\partial p_{k}}\right) \\
=\left.\left\{\dot{\xi}_{s}-\frac{\partial}{\partial p_{s}}\left[X^{(0)}(H)\right]\right\}\right|_{\dot{q}_{s}=\frac{\partial H}{\partial p_{s}}}, \\
\left(\tilde{Q}_{s}^{k}-\tilde{\nu}_{s}^{k}\right)\left(\dot{p}_{k}+\frac{\partial H}{\partial q_{k}}\right) \\
=\left.\left\{\dot{\eta}_{s}+\frac{\partial}{\partial q_{s}}\left[X^{(0)}(H)\right]\right\}\right|_{\dot{p}_{s}=-\frac{\partial H}{\partial q_{s}}}, \\
s, k=1, \ldots, n .
\end{gathered}
$$

If $\tilde{P}_{s}^{k}=\tilde{\mu}_{s}^{k}, \tilde{Q}_{s}^{k}=\tilde{\nu}_{s}^{k}$, then we have Eq. (7), which implies that the system is second-class Mei symmetrical. 


\section{Conformal factor of conformal invariance} for Hamilton system

In fact, taking

$$
\begin{aligned}
& \left\{\dot{\xi}_{s}-\frac{\partial}{\partial p_{s}}\left[X^{(0)}(H)\right]\right\} \\
& -\left.\left\{\dot{\xi}_{s}-\frac{\partial}{\partial p_{s}}\left[X^{(0)}(H)\right]\right\}\right|_{\dot{q}_{s}=\frac{\partial H}{\partial p_{s}}}, \\
& \left\{\dot{\eta}_{s}+\frac{\partial}{\partial q_{s}}\left[X^{(0)}(H)\right]\right\} \\
& -\left.\left\{\dot{\eta}_{s}+\frac{\partial}{\partial q_{s}}\left[X^{(0)}(H)\right]\right\}\right|_{\dot{p}_{s}=-\frac{\partial H}{\partial q_{s}}},
\end{aligned}
$$

we can obtain the conformal factor of conformal invariance for Hamilton system.

Using

$$
\begin{aligned}
& \dot{\xi}_{s}=\frac{\partial \xi_{s}}{\partial t}+\frac{\partial \xi_{s}}{\partial q_{k}} \dot{q}_{k}+\frac{\partial \xi_{s}}{\partial p_{k}} \dot{p}_{k}, \\
& \dot{\eta}_{s}=\frac{\partial \eta_{s}}{\partial t}+\frac{\partial \eta_{s}}{\partial q_{k}} \dot{q}_{k}+\frac{\partial \eta_{s}}{\partial p_{k}} \dot{p}_{k},
\end{aligned}
$$

we have

$$
\begin{aligned}
\dot{\xi}_{s} & -\frac{\partial}{\partial p_{s}}\left[X^{(0)}(H)\right] \\
= & \frac{\partial \xi_{s}}{\partial t}+\frac{\partial \xi_{s}}{\partial q_{k}} \dot{q}_{k}+\frac{\partial \xi_{s}}{\partial p_{k}} \dot{p}_{k} \\
& -\frac{\partial}{\partial p_{s}}\left(\xi_{0} \frac{\partial H}{\partial t}+\xi_{s} \frac{\partial H}{\partial q_{s}}+\eta_{s} \frac{\partial H}{\partial p_{s}}\right), \\
\left.\left\{\dot{\xi}_{s}-\frac{\partial}{\partial p_{s}}\left[X^{(0)}(H)\right]\right\}\right|_{\dot{q}_{s}=\frac{\partial H}{\partial p_{s}}} & =\frac{\partial \xi_{s}}{\partial t}+\frac{\partial \xi_{s}}{\partial q_{k}} \frac{\partial H}{\partial p_{k}}+\frac{\partial \xi_{s}}{\partial p_{k}} \dot{p}_{k} \\
& -\frac{\partial}{\partial p_{s}}\left(\xi_{0} \frac{\partial H}{\partial t}+\xi_{s} \frac{\partial H}{\partial q_{s}}+\eta_{s} \frac{\partial H}{\partial p_{s}}\right)
\end{aligned}
$$

therefore

$$
\begin{aligned}
& \left\{\dot{\xi}_{s}-\frac{\partial}{\partial p_{s}}\left[X^{(0)}(H)\right]\right\} \\
& \quad-\left.\left\{\dot{\xi}_{s}-\frac{\partial}{\partial p_{s}}\left[X^{(0)}(H)\right]\right\}\right|_{\dot{q}_{s}=\frac{\partial H}{\partial p_{s}}} \\
& =\frac{\partial \xi_{s}}{\partial q_{k}}\left(\dot{q}_{k}-\frac{\partial H}{\partial p_{k}}\right)=\tilde{\mu}_{s}^{k}\left(\dot{q}_{k}-\frac{\partial H}{\partial p_{k}}\right) .
\end{aligned}
$$

By the same reason, we obtain

$$
\begin{aligned}
& \left\{\dot{\eta}_{s}+\frac{\partial}{\partial q_{s}}\left[X^{(0)}(H)\right]\right\} \\
& \quad-\left.\left\{\dot{\eta}_{s}+\frac{\partial}{\partial q_{s}}\left[X^{(0)}(H)\right]\right\}\right|_{\dot{p}_{s}=-\frac{\partial H}{\partial q_{s}}} \\
& =\frac{\partial \eta_{s}}{\partial p_{k}}\left(\dot{p}_{k}+\frac{\partial H}{\partial q_{k}}\right)=\tilde{\nu}_{s}^{k}\left(\dot{p}_{k}+\frac{\partial H}{\partial q_{k}}\right) .
\end{aligned}
$$

Furthermore, we can find the conformal factor that the conformal invariance would be the second-class Mei symmetry of Hamilton system

$$
\tilde{P}_{s}^{k}=\frac{\partial \xi_{s}}{\partial q_{k}}, \quad \tilde{Q}_{s}^{k}=\frac{\partial \eta_{s}}{\partial p_{k}}, \quad s, k=1, \ldots, n .
$$

\section{Structure equation and conserved quantity of conformal invariance under second-class Mei symmetry}

The conformal invariance of Hamilton system can also lead to a conserved quantity under certain conditions.

Theorem 3. For the Hamilton system (1), if the generators $\xi_{0}, \xi_{s}$ and $\eta_{s}$ of the infinitesimal transformations (3) satisfy the determining Eqs. (7) in the second-class Mei symmetry, and there exists a gauge function $G=G(t, \boldsymbol{q}, \boldsymbol{p})$ satisfying the following Mei symmetrical structure equation:

$$
\begin{aligned}
& X^{(0)}\left(p_{s}\right) \dot{\xi}_{s}+\frac{\mathrm{d}}{\mathrm{d} t}\left\{X^{(0)}\left(p_{s}\right)\right\} \xi_{s}-X^{(0)}(H) \dot{\xi}_{0} \\
& -\frac{\partial}{\partial t}\left\{X^{(0)}(H)\right\} \xi_{0}+\left(\frac{\partial H}{\partial p_{s}} \dot{\eta}_{s}+\frac{\partial H}{\partial q_{s}} \dot{\xi}_{s}\right) \xi_{0}+\dot{G} \\
& \quad=0,
\end{aligned}
$$

then the conformal invariance of the Hamilton system (1) possesses the following conserved quantity:

$$
I=X^{(0)}\left(p_{s}\right) \xi_{s}-X^{(0)}(H) \xi_{0}+G=\text { const. }
$$

Proof. Taking the derivatives of $I$ with respect to time in Eq. (22), and using Eq. (7), (1) and (21), we can obtain

$$
\begin{aligned}
\frac{\mathrm{d} I}{\mathrm{~d} t} & =X^{(0)}\left(p_{s}\right) \dot{\xi}_{s}+\frac{\mathrm{d}}{\mathrm{d} t}\left\{X^{(0)}\left(p_{s}\right)\right\} \xi_{s}-X^{(0)}(H) \dot{\xi}_{0} \\
& -\frac{\partial}{\partial t}\left\{X^{(0)}(H)\right\} \xi_{0}-\frac{\partial}{\partial q_{s}}\left\{X^{(0)}(H)\right\} \dot{q}_{s} \xi_{0} \\
& -\frac{\partial}{\partial p_{s}}\left\{X^{(0)}(H)\right\} \dot{p}_{s} \xi_{0}+\dot{G} \\
& =X^{(0)}\left(p_{s}\right) \dot{\xi}_{s}+\frac{\mathrm{d}}{\mathrm{d} t}\left\{X^{(0)}\left(p_{s}\right)\right\} \xi_{s}-X^{(0)}(H) \dot{\xi}_{0} \\
& -\frac{\partial}{\partial t}\left\{X^{(0)}(H)\right\} \xi_{0}+\left(\dot{\eta}_{s} \frac{\partial H}{\partial p_{s}}+\dot{\xi}_{s} \frac{\partial H}{\partial q_{s}}\right) \xi_{0}+\dot{G} \\
& =0 .
\end{aligned}
$$

\section{An illustrative example}

We study a Hamilton system with two freedom degrees

$$
H=\left(p_{1} p_{2}+q_{1} q_{2}\right)^{1 / 2} \text {, }
$$

taking

$$
\text { then }
$$$$
\xi_{0}=1, \quad \xi_{1}=q_{1}, \quad \xi_{2}=q_{2},
$$$$
\eta_{1}=p_{1}, \quad \eta_{2}=p_{2},
$$

$$
X^{(0)}(H)=H,
$$


$M=1$.

According to the determining Eqs. (9), we can obtain the conformal factor of conformal invariance for the second-class Mei symmetry

$$
\tilde{P}_{s}^{k}=\tilde{Q}_{s}^{k}=\left(\begin{array}{ll}
1 & 0 \\
0 & 1
\end{array}\right) .
$$

It can also be obtained from Eq. (20). The determining equations of conformal invariance are

$$
\begin{aligned}
& \left(\begin{array}{c}
\dot{\xi}_{1}-\frac{\partial}{\partial p_{1}}\left\{X^{(0)}(H)\right\} \\
\dot{\xi}_{2}-\frac{\partial}{\partial p_{2}}\left\{X^{(0)}(H)\right\}
\end{array}\right)=\left(\begin{array}{ll}
1 & 0 \\
0 & 1
\end{array}\right)\left(\begin{array}{c}
\dot{q}_{1}-\frac{\partial H}{\partial p_{1}} \\
\dot{q}_{2}-\frac{\partial H}{\partial p_{2}}
\end{array}\right), \\
& \left(\begin{array}{c}
\dot{\eta}_{1}+\frac{\partial}{\partial q_{1}}\left\{X^{(0)}(H)\right\} \\
\dot{\eta}_{2}+\frac{\partial}{\partial q_{2}}\left\{X^{(0)}(H)\right\}
\end{array}\right)=\left(\begin{array}{ll}
1 & 0 \\
0 & 1
\end{array}\right)\left(\begin{array}{c}
\dot{p}_{1}+\frac{\partial H}{\partial q_{1}} \\
\dot{p}_{2}+\frac{\partial H}{\partial q_{2}}
\end{array}\right),
\end{aligned}
$$

then the system possesses the characteristics of both conformal invariance of second-class Mei symmetry and the second-class Mei symmetry.

From Eq. (21) we can get

$$
\eta_{s} \dot{\xi}_{s}+\dot{\eta}_{s} \xi_{s}+\dot{G}=\frac{\mathrm{d}}{\mathrm{d} t}\left(\eta_{s} \xi_{s}\right)+\dot{G}=0
$$

thus the gauge function of second-class Mei symmetry is

$$
G=-\left(\eta_{s} \xi_{s}\right)=-p_{1} q_{1}-p_{2} q_{2} \text {. }
$$

From the conserved quantity in Eq. (22), we can conclude that

$$
I=-H=-\left(p_{1} p_{2}+q_{1} q_{2}\right)^{1 / 2}=\text { const. }
$$

\section{Conclusion}

For a Hamilton system, by using the definition of second-class Mei symmetry and its conformal invariance under the single-parameter infinitesimal transformations, we can obtain the conformal factor of determining equations of conformal invariance. The conformal factor is also the necessary and sufficient condition that the conformal invariance would be Mei symmetry of the system. If the Hamilton equations possess the characteristics of conformal invariance of second-class Mei symmetry, then they possess second-class Mei symmetry. Conformal invariance of Mei symmetry can lead to a conserved quantity under certain condition as well.

\section{Acknowledgments}

Project supported by the National Natural Science Foundation of China (grant No. 10572021, 10772025).

\section{References}

[1] A.E. Noether, Nachr. Akad. Wiss. Göttingen Math. Phys. KI II, 235 (1918).
[2] Z.P. Li, Classical and Quantal Dynamics of Constrained Systems and Their Symmetry Properties, Beijing Polytechnic University Press, Beijing 1993.

[3] Z.P. Li, Constrained Hamiltonian Systems and Their Symmetry Properties, Beijing Polytechnic University Press, Beijing 1999.

[4] F.X. Mei, Symmetries and Conserved Quantities of Constrained Mechanical Systems, Beijing Institute of Technology Press, Beijing 2004.

[5] M. Lutzky, J. Phys. A 12, 973 (1979).

[6] F.X. Mei, J. Beijing Inst. Tech. 9, 120 (2000).

[7] S.K. Luo, Acta Phys. Sin. 52, 712 (2003).

[8] H. Li, J.H. Fang, Acta Phys. Sin. 53, 2807 (2004).

[9] Y. Zhang, W.K. Ge, Acta Phys. Sin. 54, 1464 (2005).

[10] Y. Zhang, Acta Phys. Sin. 54, 2980 (2005).

[11] Z.M. Lou, Acta Phys. Sin. 54, 1015 (2005).

[12] S.W. Zheng, L.Q. Jia, H.S. Yu, Chin. Phys. 15, 1399 (2006).

[13] S.L. Gu, H.B. Zhang, Acta Phys. Sin. 55, 5594 (2006).

[14] W.K. Ge, Acta Phys. Sin. 56, 1 (2007).

[15] S.W. Zheng, L.Q. Jia, Acta Phys. Sin. 56, 661 (2007).

[16] Y. Zhang, Y. Xue, Acta Phys. Sin. 50, 816 (2001).

[17] S.K. Luo, L.Q. Jia, J.L. Cai, Chin. Phys. 12, 841 (2003).

[18] S.K. Luo, L.Q. Jia, Commun. Theor. Phys. 40, 265 (2003).

[19] F.X. Mei, Acta Phys. Sin. 52, 1048 (2003).

[20] Y. Zhang, Acta Phys. Sin. 52, 1326 (2003).

[21] J.L. Fu, L.Q. Chen, Mech. Res. Commun. 31, 9 (2004).

[22] S.K. Luo, Acta Phys. Sin. 53, 5 (2004).

[23] R.W. Liu, L.Q. Chen, Chin. Phys. 13, 1615 (2004).

[24] J.L. Fu, L.Q. Chen, F.P. Xie, Chin. Phys. 13, 1611 (2004).

[25] S.K. Luo, Acta Phys. Sin. 52, 1 (2003).

[26] J.H. Fang, Y. Peng, Y.P. Liao, Acta Phys. Sin. 54, 496 (2005).

[27] J.H. Fang, Y.P. Liao, Y. Peng, Acta Phys. Sin. 54, 500 (2005).

[28] L.Q. Jia, S.W. Zheng, Acta Phys. Sin. 55, 3829 (2006).

[29] J.H. Fang, N. Ding, P. Wang, Acta Phys. Sin. 56, 3039 (2007)

[30] J.L. Cai, F.X. Mei, Acta Phys. Sin. 57, 5369 (2008).

[31] J.L. Cai, Chin. Phys. Lett. 25, 1523 (2008).

[32] J.L. Cai, S.K. Luo, F.X. Mei, Chin. Phys. B 17, 3170 (2008)

[33] J.L. Cai, Acta Phys. Pol. A 115, 854 (2009).

[34] J.L. Cai, Acta Phys. Sin. 58, 22 (2009). 